\title{
Causal Entropy Bound for a Spacelike Region
}

\author{
R. Brustein ${ }^{1}$ and G. Veneziano ${ }^{2}$ \\ ${ }^{1}$ Department of Physics, Ben-Gurion University, Beer-Sheva 84105, Israel \\ ${ }^{2}$ Theory Division, CERN, CH-1211, Geneva 23, Switzerland \\ (Received 7 December 1999; revised manuscript received 14 February 2000)
}

\begin{abstract}
The identification of a causal-connection scale motivates us to propose a new covariant bound on entropy within a generic spacelike region. This "causal entropy bound," scaling as $\sqrt{E V}$, and thus lying around the geometric mean of Bekenstein's $S / E R$ and holographic $S / A$ bounds, is checked in various "critical" situations. In the case of limited gravity, Bekenstein's bound is the strongest while naive holography is the weakest. In the case of strong gravity, our bound and Bousso's holographic bound are stronger than Bekenstein's, while naive holography is too tight, and hence typically wrong.
\end{abstract}

PACS numbers: 04.70.Dy, 04.20.Gz

The second law of thermodynamics states that the entropy of a closed system tends to grow towards its largest possible value. But what is this maximal value? Bekenstein [1] has suggested that, for a limited gravity system of energy $E$, whose size $R$ is larger than its gravitational radius, $R>R_{g} \equiv 2 G_{N} E$, entropy is bounded by $S_{\mathrm{BEB}}$,

$$
S_{\mathrm{BEB}}=E R / \hbar=R_{g} R l_{P}^{-2},
$$

where $l_{P}$ is the Planck length (throughout this paper we will stress functional dependence, while ignoring numerical factors, and set $c=k_{B}=1$ ). Note that (1) bounds the ratio entropy/energy for a system of given size. The entropy of normal systems, such as matter in thermal equilibrium, is well below bound (1), moreover, in the 18 years which elapsed since Bekenstein's proposal, and despite an ongoing debate [2], no physical example in which (1) is violated has been produced. Is the same bound applicable to more general situations, for example, in cosmology? This question was addressed by Bekenstein himself [3], who gave a prescription for a cosmological extension by choosing $R$ in Eq. (1) to be the particle horizon. Is this a correct extension? And, even if so, is it possible to find stronger bounds for systems that are not of limited gravity?

Holography [4] suggests that maximal entropy is bounded by $S_{\mathrm{HOL}}$,

$$
S_{\mathrm{HOL}}=A l_{P}^{-2},
$$

where $A$ is the area of the spacelike surface enclosing a certain region of space. For systems of limited gravity, since $R>R_{g}, A=R^{2}$, (1) implies the holography bound (2). Is it possible to extend entropy/area holographic bounds to more general situations, for example, to cosmology where, for large enough regions, it soon becomes tighter than entropy/energy bounds? This issue was first addressed by Fischler and Susskind (FS) [5], who proposed that the area of the particle horizon should bound the entropy on the backward-looking light cone according to (2). It was soon realized, however, that the FS proposal requires modifica- tions, since violations of it were found to occur in physically reasonable situations.

Several attempts were made to mend the FS proposal, first within cosmology [6-9], and then, by Bousso, in arbitrary space-times [10]. In some cosmological situations, Bousso's proposal reduces to the previously proposed ones, which identify the maximal size of a spatial region for which holography works with the Hubble radius [6,7] (or apparent horizon [8]), while in other situations it is quite different. The advantages of Bousso's proposal are that (i) it is very general; and (ii) it is manifestly covariant. A possible shortcoming of the proposal is that it bounds entropy on lightlike hypersurfaces: in order to extend the bound to spacelike regions, a "spacelike projection," which is not always possible, has to be performed [10].

We make here a motivated proposal for an improved covariant bound, applicable to entropy on spacelike hypersurfaces, and test it in several critical cases. We then compare our bound to other proposals, in particular to Bekenstein's and Bousso's, and show that, for systems of limited gravity, Bekenstein's bound is the tightest, while, in other situations, our bound is the strongest one proposed so far which does not lead to contradictions for spacelike regions. A crucial difference between our proposal and Bousso's is that Bousso decides from the start to look for a holographic $S / A$ bound and, as he points out, this forces him to consider lightlike hypersurfaces. We do not insist, a priori, on a holographic bound, but aim at generality of the hypersurface and check how holography may or may not work a posteriori.

As in the previous proposals, we shall refer to entropy in a region as to a quantity proportional to the number of degrees of freedom in that region. To be more precise, we shall exclude from consideration entropy associated with the gravitational field itself, that we treat here classically. Let us first state our proposal, and then motivate and test it. Consider a generic spacelike hypersurface, defined by the equation $\tau=0$, and a compact region lying within it defined by $\sigma \leq 0$. We propose that the entropy contained in this region, $S(\tau=0, \sigma \leq 0)$, is bounded by $S_{\mathrm{CEB}}$, 


$$
\begin{aligned}
S_{\mathrm{CEB}} & =l_{P}^{-2} \int_{\sigma<0} d^{4} x \sqrt{-g} \delta(\tau) \sqrt{\max _{ \pm}\left[\left(G_{\mu \nu} \pm R_{\mu \nu}\right) \partial^{\mu} \tau \partial^{\nu} \tau\right]} \\
& =l_{P}^{-1} \hbar^{-1 / 2} \int_{\sigma<0} d^{4} x \sqrt{-g} \delta(\tau) \sqrt{\max _{ \pm}\left[\left(T_{\mu \nu} \pm T_{\mu \nu} \mp \frac{1}{2} g_{\mu \nu} T\right) \partial^{\mu} \tau \partial^{\nu} \tau\right]}
\end{aligned}
$$

Here $G_{\mu \nu}, R_{\mu \nu}$ are the Einstein and Ricci tensor, respectively, $T_{\mu \nu}$ is the energy-momentum tensor, and $T$ its trace. To derive the second equality we have used Einstein's equations, $G_{\mu \nu}=8 \pi G_{N} T_{\mu \nu}$. Note the appearance of the square root of the energy contained in the region, which we alluded to in the abstract. Note also that (3) is manifestly covariant, and invariant under reparametrization of the hypersurface equation: such an invariance requires a square root of $\partial^{\mu} \tau \partial^{\nu} \tau$. Reality of $S_{\mathrm{CEB}}$ is assured if sources obey the weak energy condition, $T_{\mu \nu} \partial^{\mu} \tau \partial^{\nu} \tau \geq 0$, since then the sum of the two combinations in (3), and thus their maximum, are positive. The weak energy condition is sufficient, but not necessary, for reality. We expect that, for physical systems, reality will be always guaranteed [see also the comments after Eq. (8)].

Since (3) applies to any spacelike region, it can be written in a local, rather than integrated, form by introducing an entropy current $s_{\mu}$ such that $S=\int d^{4} x \sqrt{-g} \delta(\tau) s_{\mu} \partial^{\mu} \tau$. Then (3) becomes equivalent to $\left(\lambda^{\mu}\right.$ being an arbitrary timelike vector):

$$
\begin{aligned}
s_{\mu} \lambda^{\mu} \leq & l_{P}^{-1} \hbar^{-1 / 2} \\
& \times \sqrt{\max _{ \pm}\left[\left(T_{\mu \nu} \pm T_{\mu \nu} \mp \frac{1}{2} g_{\mu \nu} T\right) \lambda^{\mu} \lambda^{\nu}\right]}
\end{aligned}
$$

In the limit in which the hypersurface is lightlike, $\partial^{\mu} \tau \partial_{\mu} \tau=0$, Eqs. (3) and (4) read

$$
\begin{aligned}
& S_{\mathrm{CEB}}=\int_{\sigma<0} d^{4} x \sqrt{-g} \delta(\tau) \sqrt{T_{\mu \nu} \partial^{\mu} \tau \partial^{\nu} \tau}, \\
& s_{\mu} \lambda^{\mu} \leq l_{P}^{-1} \hbar^{-1 / 2} \sqrt{T_{\mu \nu} \lambda^{\mu} \lambda^{\nu}}, \quad \lambda_{\mu} \lambda^{\mu}=0,
\end{aligned}
$$

and become closely related to the assumptions made in [11] [Eq. (1.10)] in order to derive a modified Boussotype bound.

The physical motivations leading us to the above proposal are similar to those used in the recently proposed Hubble-entropy-bound (HEB) [7] (see also [6,8,9]), i.e., (i) that entropy is maximized, in a given region of space, by the largest black hole that can fit in it; (ii) that the largest black hole that can hold together without falling apart in a cosmological background has typically the size of the Hubble horizon. This second, crucial assumption appears to be supported qualitatively by a number of previous results [12], but clearly needs to be refined and, possibly, to be defined covariantly. With such a goal in mind, we will proceed as follows: we will start by identifying a critical ("Jeans") length scale above which perturbations are causally disconnected so that black holes of larger size, very likely, cannot form. We will first find this causal connection (CC) scale $R_{\mathrm{CC}}$ for the simplest cosmological backgrounds, then extend it to more general cases and, finally, guess the completely general expression using general covariance. In other words, we shall be using cosmological models as a theoretical laboratory in which physical arguments about the maximal size of classically stable black holes lead to an interesting bound on the largest region for which holography can work.

In order to identify the CC scale for a homogeneous, isotropic, and spatially flat background, let us consider a generic perturbation around such a background in the Hamiltonian approach developed in [13]. The Fourier components of the (normalized) perturbation and of its (normalized) conjugate momentum satisfy Schrödingerlike equations $\hat{\Psi}_{k}^{\prime \prime}+\left[k^{2}-\left(S^{1 / 2}\right)^{\prime \prime} S^{-1 / 2}\right] \hat{\Psi}_{k}=0, \hat{\Pi}_{k}^{\prime \prime}+$ $\left[k^{2}-\left(S^{-1 / 2}\right)^{\prime \prime} S^{1 / 2}\right] \hat{\Pi}_{k}=0$, where $k$ is the comoving momentum, a prime denotes differentiation with respect to conformal time $\eta$, and $S^{1 / 2}$ is the so-called "pump field," a combination of the various backgrounds which depends on the specific perturbation under study. The perturbation equations clearly identify a "Jeans-like" CC comoving momentum

$$
\begin{aligned}
k_{\mathrm{CC}}^{2} & =\max \left[\left(S^{1 / 2}\right)^{\prime \prime} S^{-1 / 2},\left(S^{-1 / 2}\right)^{\prime \prime} S^{1 / 2}\right] \\
& =\max \left[\mathcal{K}^{\prime}+\mathcal{K}^{2},-\mathcal{K}^{\prime}+\mathcal{K}^{2}\right],
\end{aligned}
$$

where $\mathcal{K}=\left(S^{1 / 2}\right)^{\prime} S^{-1 / 2}$. Note that Eq. (6) always defines a real $k_{\mathrm{CC}}$ since the sum of the two quantities appearing on the right-hand side (rhs) is positive semidefinite. Since tensor perturbations are always present, let us restrict our attention to them. The "pump field" $S^{1 / 2}$ is simply given, in this case, by the scale factor $a(\eta)$ so that $\mathcal{K} \rightarrow \mathcal{H}=a^{\prime} / a$. Equation (6) is immediately converted into the definition of a proper "Jeans" CC length $R_{\mathrm{CC}}=a k_{\mathrm{CC}}^{-1}$. Substituting into Eq. (6), and expressing the result in terms of proper-time quantities, we obtain (for tensor perturbations) $R_{\mathrm{CC}}^{-2}=\max \left[\dot{H}+2 H^{2},-\dot{H}\right]$. Before trying to recast this equation in a more covariant form let us remove the assumption of spatial flatness by introducing the usual spatial-curvature parameter $\kappa$ $(\kappa=0, \pm 1)$. The study of perturbations in nonflat space [14] is considerably more complicated than in a spatially flat background. The final result, however, appears to be extremely simple [15], and can be obtained from the flat case by the following replacements in Eq. (6): $\mathcal{H}^{2} \rightarrow \mathcal{H}^{2}+\kappa, \mathcal{H}^{\prime} \rightarrow \mathcal{H}^{\prime}$. Using this simple rule (see below for another confirmation of its validity) we arrive at the following generalization: 


$$
R_{\mathrm{CC}}^{-2}=\max \left[\dot{H}+2 H^{2}+\kappa / a^{2},-\dot{H}+\kappa / a^{2}\right] .
$$

At this point we could have introduced anisotropy in our homogeneous background and study perturbations with or without spatial curvature. This should certainly be done as a check of a shortcut procedure based on general covariance that we adopt instead. We note that the 00 components of the Ricci and Einstein tensors for our background are given by $R_{00}=-3\left(\dot{H}+H^{2}\right), G_{00}=3\left(H^{2}+\kappa / a^{2}\right)$. Obviously,

$$
\begin{aligned}
R_{\mathrm{CC}}^{-2} & =\frac{1}{3} \max _{\mp}\left[G_{00} \mp R_{00}\right] \\
& =4 \pi G_{N} \max \left[\frac{\rho}{3}-p, \rho+p\right],
\end{aligned}
$$

where we have inserted Einstein's equations using, as an example, a perfect-fluid energy momentum tensor, $T_{\nu}^{\mu}=$ $\operatorname{diag}(\rho,-p,-p,-p)$. Equation (8) is guaranteed to define a real $R_{\mathrm{CC}}$ if the weak energy condition (reading here $\rho>0$ ) holds, since the sum of the two combinations is positive in this case. In general, other perturbations may compete with tensor perturbations and define a smaller $R_{\mathrm{CC}}$. In this case, the symbol "max" in the above equations also applies to the various types of perturbations. This may help to ensure reality of $R_{\mathrm{CC}}$ in all physical situations.

As a final step, let us convert Eq. (8) into an explicitly covariant bound on entropy using, as in [7], the idea that entropy is maximized by having maximal size black holes filling up the volume. Using $R_{\mathrm{CC}}$ as the maximal scale for black holes, we get a bound on entropy which scales like $S \sim V R_{\mathrm{CC}}^{-3} R_{\mathrm{CC}}^{2} l_{P}^{-2}=V R_{\mathrm{CC}}^{-1} l_{P}^{-2}$. We now express $R_{\mathrm{CC}}^{-1}$ as in (8) in terms of the components of the Ricci and Einstein tensors in the direction orthogonal to the hypersurface on which the entropy is being computed. This can be done covariantly by defining the hypersurface through the equation $\tau=0$ and by identifying the normal with the vector $\nabla^{\mu} \tau$. This procedure leads immediately to the proposal (3). The local form (4) clearly follows by shrinking the spacelike region to a point. Alternatively, using standard $3+1$ ADM formalism [16], we can express the relevant components of the Ricci and Einstein tensors in terms of the intrinsic and extrinsic curvature of the hypersurface under study.

We turn to check our proposal for various physical systems and to verify that it is sensible.

(1) Systems of limited gravity. - We note (see below) that for systems of limited gravity the Bekenstein bound is tighter than ours $S_{\mathrm{BEB}}<S_{\mathrm{CEB}}$. Therefore, in all systems for which the BEB is obeyed, ours will be obeyed as well.

(2) Cosmology. - The universe is a system of strong self-gravity. The geometry of the universe is determined by self-gravity, and the size of the universe is at least its gravitational radius. The strongest challenges to entropy bounds in general, and to our bound in particular, come from considering (re)collapsing universes.

We discuss three cases basically exhausting the possible types of cosmologies:

(i) $|\dot{H}| \sim H^{2}$ : In this region effective energy density and pressure are of the same order, $\rho \sim p$, and all previously suggested length scales that should be considered in entropy bounds, such as particle horizon, apparent horizon, $R_{\mathrm{CC}}$, Hubble length, are parametrically equal. In particular, it is already established that HEB is not violated if some reasonable restrictions on the equation of state are imposed, and therefore our bound (and others) is also valid.

(ii) $|\dot{H}| \ll H^{2}$ : In this case $|\rho+p| \ll \rho$, and the universe is inflationary. Here the naive holographic bound fails miserably, but HEB does well. Since in this case $R_{\mathrm{CC}}$ is parametrically equal to $|H|^{-1}$, it follows that CEB works as well as HEB.

(iii) $|\dot{H}| \gg H^{2}$, i.e., $|\rho| \ll p$ : Since $\rho$ and $p$ are the effective energy density and pressure, there are no problems with causality. This case occurs, for instance, near the turning point of an expanding universe which recollapses as the result of a negative cosmological constant, of positive spatial curvature (or of both). Both the naive HEB and the apparent horizon bound (AHB) of [8] fail here, while Bousso's prescription does fine. We would like to show that CEB can easily cope with this third case.

Consider either a flat or closed universe with some perfect fluid in thermal equilibrium and a constant equation of state $p=\gamma \rho, 1>\gamma>-1$, and with an additional small negative cosmological constant $\Lambda=-\lambda$. The universe starts out expanding, reaches a maximal size, and then contracts towards a singularity. In this case, matter entropy within a comoving volume is constant in time, but near the point of maximal expansion the apparent horizon, and the Hubble length, diverge, causing violation of the HEB and AHB. However, for a fixed comoving volume, $S_{\mathrm{CEB}} \sim a^{3} R_{\mathrm{CC}}^{-1}$, and, since $R_{\mathrm{CC}}$ is never larger than some maximal value, CEB has a chance of doing better.

To see this explicitly, note that, in this case, and independently of $\kappa, R_{\mathrm{CC}}^{-2}=(1 / 3) \max \left[1 / 2 \rho_{0}(1-3 \gamma) \times\right.$ $\left.a^{-3(1+\gamma)}-2 \lambda,(3 / 2)(1+\gamma) \rho_{0} a^{-3(1+\gamma)}\right] \geq(1 / 2) \times$ $(1+\gamma) \rho_{0} a^{-3(1+\gamma)}$, where $\rho_{0}$ is the initial energy density and $a$ is the ratio of the scale factor to its initial value. It follows that, in a fixed comoving volume, $S_{\mathrm{CEB}} \sim a^{3 / 2(1-\gamma)}$. Since $\gamma<1$, this means that $S_{\mathrm{CEB}}$ grows during the expansion, reaches a maximum at the turning point, and then starts decreasing. If we give initial conditions at a time when curvature and cosmological constant are negligible, which is always the case at sufficiently early times, CEB will be obeyed initially provided energy density and curvature are less than Planckian. But then the above evolution of $S_{\mathrm{CEB}}$ will guarantee that the bound is fulfilled at all times until Planckian density and curvature is reached in the recollapsing phase, i.e., throughout the classical evolution of our Universe. 
(3) Collapsing regions. - In this case we have limited computational power. While the local form (4) looks most appropriate for the study of collapsing regions, most likely the analysis of the general case will need the use of numerical methods. We can qualitatively check cases that are similar to the cosmological ones [17], such as homogeneous, isotropic contracting pressureless regions, or a contracting homogeneous, isotropic region filled with a perfect fluid. The pressureless case can be described by a Friedman interior and a Schwarzschild exterior. Since $\mathrm{CEB}$ is valid for the analog cosmological solution it is also valid for this case.

A particularly interesting case is that of the (generically nonhomogeneous) collapse of a stiff fluid ( $p=\rho$ ), which, up to a simple field redefinition, can be mapped onto the dilaton-driven inflation of string cosmology [18]. In this case one finds a constant $S_{\text {CEB }}$ in agreement with the HEB result [7]. Hence, no problem arises in this case, even if one starts from a saturated $S_{\mathrm{CEB}}$ at the onset of collapse. For nonstiff equations of state, the situation appears less safe if one starts near saturation (this was already pointed out in [10] for Bousso's case).

Finally, we compare our CEB to previously proposed bounds, in particular to Bekenstein's and Bousso's. For systems of limited gravity whose size exceeds their Schwarzschild radius: $R>R_{g}$, Bekenstein's bound is given by $S<S_{\mathrm{BEB}}=l_{P}^{-2} R R_{g}$, and Bousso's procedure results in the holography bound (2) [10], $S<S_{\mathrm{HOL}}=$ $l_{P}^{-2} R^{2}$, but since $R>R_{g}, S_{\mathrm{BEB}}<S_{\mathrm{HOL}}$, and therefore Bousso's bound is less stringent than Bekenstein's. Consider now CEB applied to the region of size $R$ containing an isolated system. Expressing CEB in the form (3) one immediately obtains: $S_{\mathrm{CEB}}=l_{P}^{-1} R^{3 / 2} E^{1 / 2} \hbar^{-1 / 2}=$ $\left(S_{\mathrm{HOL}} S_{\mathrm{BEB}}\right)^{1 / 2}$, implying $S_{\mathrm{BEB}} \leq S_{\mathrm{CEB}} \leq S_{\mathrm{HOL}}$. We conclude that for isolated systems of limited self-gravity the Bekenstein bound is the tightest, followed by our CEB and, finally, by Bousso's holographic bound. Similar scaling properties for the HEB were discussed in [7]. The same scaling laws also follow from (apparently unrelated) quantum measurement arguments [19].

For regions of space that contain so much energy that the corresponding gravitational radius $R_{g}$ exceeds $R$, Bekenstein's bound is the weakest, while the naive holography bound is the strongest (but very often wrong). Bousso's proposal (see also [8]) uses the apparent horizon $R_{\mathrm{AH}}$ while CEB uses $R_{\mathrm{CC}}$. For homogeneous cosmologies, $R_{\mathrm{CC}}<R_{\mathrm{AH}}$, since $R_{\mathrm{CC}}^{-2}$, according to (7), is always larger than the average of the two terms appearing on its rhs, which is precisely $R_{\mathrm{AH}}^{-2}=H^{2}+\kappa / a^{2}$. Since, for a fixed volume, the bounds scale like $R_{\mathrm{AH}}^{-1}$ or $R_{\mathrm{CC}}^{-1}$, we immediately find that CEB is generally more generous than the AHB proposed in [8]. This is what makes it possible for CEB to be fulfilled in the negative cosmological case discussed above, where AHB or HEB are violated. Comparison with Bousso's proposal is more subtle, since he makes use of the AH area to bound entropy on light sheets. This can be converted into a bound on the entropy of the spacelike region inside the $\mathrm{AH}$ only in special cases. That is what makes Bousso's bound fulfilled even when the straight AHB fails.

We believe that CEB, particularly in its local form (4), stands out in terms of its physical motivations and its potential for practical uses. The fact that, in its local form (4), the bound appears very sensible for subplanckian densities/curvatures, reinforces the belief that our assumptions about the maximal size of black holes make sense, and vice versa. CEB may be converted into a new kind of generalized second law following [20], and used to study cosmological singularities.

We thank J. Bekenstein, R. Bousso, M. Gasperini, N. Kaloper, G. Kane, A. Linde, R. Madden, and R. Wald for comments, suggestions, criticism, and useful discussions, and N. Sasakura for informing us about his work. R. B. thanks CERN TH division for hospitality.

[1] J. D. Bekenstein, Phys. Rev. D 23, 287 (1981); 49, 1912 (1994), and references therein.

[2] For a recent overview, see J. D. Bekenstein, Phys. Rev. D 60, 124010 (1999).

[3] J. D. Bekenstein, Int. J. Theor. Phys. 28, 967 (1989).

[4] G.'t Hooft, Abdus Salam Festschrift: A Collection of Talks, edited by A. Ali, J. Ellis, and S. Randjbar-Daemi (World Scientific, Singapore, 1993); L. Susskind, J. Math. Phys. 36, 6377 (1995), and references therein.

[5] W. Fischler and L. Susskind, hep-th/9806039.

[6] R. Easther and D. A. Lowe, Phys. Rev. Lett. 82, 4967 (1999).

[7] G. Veneziano, Phys. Lett. B 454, 22 (1999); hep-th/ 9907012.

[8] D. Bak and S. Rey, hep-th/9902173.

[9] N. Kaloper and A. Linde, Phys. Rev. D 60, 103509 (1999).

[10] R. Bousso, JHEP 7, 4 (1999); 6, 28 (1999); hep-th/ 9911002.

[11] E.E. Flanagan, D. Marolf, and R.M. Wald, hep-th/ 9908070.

[12] B. J. Carr and S. W. Hawking, Mon. Not. R. Astron. Soc. 168, 399 (1974); B. J. Carr, Astrophys. J. 201, 1 (1975); I. D. Novikov and A. G. Polnarev, Astron. Zh. 57, 250 (1980) [Sov. Astron. 24, 147 (1980)].

[13] R. Brustein, M. Gasperini, and G. Veneziano, Phys. Lett. B 431, 277 (1998).

[14] J. Garriga et al., Nucl. Phys. B513, 343 (1998).

[15] A. Ghosh, G. Pollifrone, and G. Veneziano, Phys. Lett. B 440, 20 (1998).

[16] See, e.g., C. W. Misner, K. S. Thorne, and J. A. Wheeler, Gravitation (Freeman, San Francisco, 1970).

[17] See Ref. [16], pp. 851-859.

[18] A. Buonanno, T. Damour, and G. Veneziano, Nucl. Phys. B543, 275 (1999).

[19] N. Sasakura, Prog. Theor. Phys. 102, 169 (1999).

[20] R. Brustein, Phys. Rev. Lett. 84, 2072 (2000); R. Brustein, S. Foffa, and R. Sturani, Phys. Lett. B 471, 352 (2000). 